Linguistique, littérature, didactique

\title{
Pour une éthique de la grammaire ou quand l'exception infirme la règle
}

For an Ethics of Grammar, or when the Exception invalidates the Rule

\section{Marc Wilmet}

\section{(2) OpenEdition}

\section{Journals}

\section{Édition électronique}

URL : http://journals.openedition.org/pratiques/2616

DOI : 10.4000/pratiques.2616

ISSN : 2425-2042

\section{Éditeur}

Centre de recherche sur les médiations (CREM)

\section{Référence électronique}

Marc Wilmet, «Pour une éthique de la grammaire ou quand l'exception infirme la règle », Pratiques [En ligne], 167-168 | 2015, mis en ligne le 01 avril 2016, consulté le 30 avril 2019. URL : http:// journals.openedition.org/pratiques/2616; DOI : 10.4000/pratiques.2616

Ce document a été généré automatiquement le 30 avril 2019.

(c) Tous droits réservés 


\title{
Pour une éthique de la grammaire ou quand l'exception infirme la règle
}

For an Ethics of Grammar, or when the Exception invalidates the Rule

\author{
Marc Wilmet
}

«Las de nourrir un obscur pensionnaire, on me
met un jour dans la rue ; et comme il faut dîner,
quoiqu'on ne soit plus en prison, je taille encore
ma plume et demande à chacun de quoi il est
question : on me dit que, pendant ma retraite
économique, il s'est établi dans Madrid un système
de liberté sur la vente des productions, qui s'étend
même à celles de la presse ; et que, pourvu que je
ne parle en mes écrits ni de l'autorité, ni du culte,
ni de la politique, ni de la morale, ni des gens en
place, ni des corps en crédit, ni de l'Opéra, ni des
autres spectacles, ni de personne qui tienne à
quelque chose, je puis tout imprimer librement,
sous l'inspection de deux ou trois censeurs. "
(Beaumarchais, Le Mariage de Figaro, V, 3)

1 Quand les organisateurs m'ont fait l'honneur - et le plaisir, évidemment - de me convier à prendre la parole en hors-d'œuvre de ce colloque consacré à l'exception, à l'exclusion et/ ou à la restriction, j'ai songé in petto que la première anomalie, bizarrerie, particularité ou singularité - au choix - était peut-être l'attention qu'ils continuent ainsi à manifester publiquement à leur ancien professeur (filiation directe pour Laurence Rosier et Dan Van Raemdonck, indirecte pour Audrey Roig). Il me reste, lourde tâche, à ne pas décevoir leur confiance*.

2 N'insistons pas sur l'écueil initial (d'autres en reparleront vraisemblablement... et moi aussi), celui des rapports trompeurs entre la langue « de tous les jours » et le vocabulaire technique des spécialistes. On sait les dégâts qu'ont causés, et que causent toujours en grammaire française, une série d'étiquettes dont beaucoup de descripteurs prétendent 
dériver les contenus: l'article "défini » définirait; l'adjectif « qualificatif» qualifierait; l'adjectif « démonstratif » montrerait à défaut de démontrer, etc. Je constate simplement, pour vite abandonner cette matière aux philosophes et aux psychologues, que le champ sémantique de la restriction ne touche guère qu'à la diminution (compression, économie, privation...), que celui de l'exception, déjà plus large, s'étend du quantitatif au qualitatif, et que le domaine de l'exclusion se ramifie à l'enseigne du verbe exclure en écarter, éliminer, évincer, proscrire, refuser, rejeter, répudier, etc. (bannir, décliner, dédaigner, ostraciser, rabrouer, radier, rebuter, récuser, refouler, rembarrer, remiser, repousser, supplanter ... -j'en oublie à coup sûr).

3 Redevenons linguistes. Le réflexe, dès l'invitation reçue et acceptée, a été d'aller chercher dans ma bibliothèque l'ouvrage de Gérard Moignet sur Les Signes de l'exception dans l'histoire du français (la thèse complémentaire du linguiste guillaumien, soutenue en 1957, publiée en 1958, rééditée avec quelques remaniements en 1973, un volume qu'il m'a offert à l'occasion du colloque Grammaire générative et psychomécanique du langage que j'avais mis sur pied la même année, et dont je relis avec émotion la dédicace : «À Marc Wilmet, en témoignage d'estime, en hommage cordial»). Je croyais ce livre tout à fait oublié (les jeunes linguistes, à l'inverse de Kronos qui dévorait ses enfants, ne se nourrissent plus guère de leurs devanciers), mais j'ai eu l'heureuse surprise de le trouver cité et exploité dans le bel article qu'Audrey Roig et Laurence Rosier (2013) ont consacré à mis à part. Grâces leur en soient rendues une deuxième fois !

G. Moignet s'intéressait principalement à la construction exceptive ne... que (avant lui, J. Damourette et É. Pichon avaient distingué un ne "discordantiel », un pas ou un point "forclusif» et un que "exceptif»). Son problème de départ: pourquoi le français classique utilisait-il simultanément le "forclusif» et l'« exceptif ", à l'image du vieil Horace de Corneille : « La gloire de leur mort m'a payé de leur perte : / Ce bonheur a suivi leur courage invaincu, / Qu'ils ont vu Rome libre autant qu'ils ont vécu, / Et ne l'auront point vue obéir qu'à son prince, / Ni d'un État voisin devenir la province »? Et pourquoi aujourd'hui le "forclusif» nie-t-il l'« exceptif»: Pierre n'aime que Marie = «Marie et personne d'autre» VS Pierre n'aime pas que Marie = "Marie et au moins quelqu'un d'autre »? Accessoirement, le fait que l'approbation d'une négation exceptive se fasse jusqu'en français classique au moyen de non (par exemple, Molière, Le Bourgeois gentilhomme : «- Il n'y a que la prose et les vers? - Non, monsieur. Tout ce qui n'est point prose est vers, et tout ce qui n'est point vers est prose ») prouve que dans cette négation en deux temps : $1^{\circ} \mathrm{du}+\mathrm{au}-, 2^{\circ} \mathrm{du}-\mathrm{au}+$, le versant négatif a longtemps dominé le versant positif : «- Ne vient-il que demain? - Non, que demain » (Marivaux), etc.

5 Autour de ne... que, de nouveaux problèmes surgissent :

- l'exception du verbe nécessite le verbe «vicaire »: On ne fera qu'en rire... Celle du sujet a besoin de la voie impersonnelle: Il n'y a que Maille qui m'aille (en raccourci familier: Que Maille qui m'aille) ou d'un pronom positif-négatif nul ou rien provoquant le basculement de ne et que en que et ne: «Nul que Mathias ne comprend pourquoi les femmes ce soir ont si longtemps promené leur ardeur inquiète » (Limbour) ou "Rien que la mort n'était capable / D'expier son forfait » (La Fontaine). Tous ces jeux autour du positif et du négatif sont loin d'avoir livré leurs secrets et mériteraient une monographie ou, pourquoi pas, une thèse. Comparer ainsi, au départ de Pierre est courageux : Pierre n'est que courageux = «le courage est son unique qualité ", à peu près équivalent à Pierre n'est rien que courageux et le contraire de Pierre est moins que courageux = « il n'arrive pas au niveau du courage », tandis que Pierre est rien moins que courageux égale « son courage ne fait aucun doute» et que sa négation Pierre 
n'est rien moins que courageux hésite entre la signification précédente ( = «il est vraiment courageux ») et son opposé ( = «il est si peu courageux qu'on ne saurait lui trouver de qualité moindre »);

- Naoyo Furukawa a levé un lièvre supplémentaire (communication personnelle). Les sousphrases pronominales sous la dépendance d'un ne... que matriciel montrent une certaine propension à délaisser la personne grammaticale 1 ou $2 \mathrm{du}$ pronom personnel au profit de la personne $3 \mathrm{du}$ verbe : «Il n'y a que toi, ma belle, qui puisse sauver Stéphanie » (Balzac). « Il n'y a que moi au monde, je le sais bien, qui soit capable d'une chose aussi extraordinaire » (Romains). Et, sans l'excuse de l'homophonie : «Y a qu'moi, madame, qui l'a vu » (Benjamin). «Y a pas qu'nous qui gueulent» (Marchand). Vulgarisme? Mais, cette fois sans soupçon de familiarité : «Il n'y a qu'elles et moi qui ne s'enrichissent pas ici» (Mille). Serait-ce que l'élément ou les éléments isolés de l'ensemble s'effacent derrière les éléments résiduels : « Il n'y a qu'elles et moi qui ne s'enrichissent pas ici » = « tous les autres s'enrichissent »?

6 Autre question jadis posée par G. Moignet, plus grammaticale que sémantique : quelle est la nature de que? Un adverbe? Encore faudrait-il se mettre d'accord sur ce qu'est un adverbe : la fonction adverbiale peut être exercée par des mots de différentes natures. Un pronom? Une conjonction? G. Moignet est sans doute un des premiers - si pas le premier - à rompre ce que j'ai appelé « la conspiration du silence » relative aux que issus en français des latins quem, quam, quod, quid ou quia, traités par les grammaires françaises comme des homonymes alors qu'on serait fondé à y trouver plutôt une organisation polysémique. L'idée pointe chez A. Lemaréchal (1989: 185) : «Traditionnellement, que complétif est considéré comme n'ayant aucun rapport en synchronie avec que relatif. Certains emplois de que établissent pourtant un pont entre les deux séries d'emplois ", puis chez P. Le Goffic (1993 : 543) : «Il faut reconnaître que le que complétif est encore sensiblement plus proche de ses origines relatives [...] qu'on ne le dit d'ordinaire ». Voyez en effet, par exemple, Pierre est plus grand que ne l'est Marie. Une prédication Pierre est grand et plus que ne l'est Marie complément de cette prédication. En tant que comparatif, que réfère à l'idée de grandeur commune à Marie et à Pierre. Il serait donc représentant, c'està-dire pronom. Or, il insère aussi une sous-phrase à l'intérieur d'une phrase matrice. Il sera donc enchâsseur et en l'occurrence conjonction. Le que de Pierre n'aime que Marie cesse d'être un enchâsseur (la phrase est simple et non complexe) mais reste un représentant (Marie est comparée à tout ce qui n'est pas elle, l'exception " Marie » se doublant d'une exclusion des « non-Marie »).

7 Je crains fort que ces spéculations ne soient plus très à la mode parmi les linguistes (il n'y a qu'à voir la désaffection des sections Syntaxe dans de grands congrès comme celui de la Société de linguistique romane ou le Congrès mondial de linguistique française). Les ressources de la Toile ont mis ou remis en selle une linguistique des corpus. Tous les «signes d'exception» que répertorie G. Moignet appellent dorénavant des enquêtes approfondies, variant les cotextes et les contextes. En plusieurs pans :

1. les vieillis fors et fors que (ne devant guère leur survie qu'à la paraphrase « Tout est perdu fors l'honneur » du passage d'une lettre de François Ir à sa mère après la bataille de Pavie : «De toute chose ne m'est demeuré que l'honneur et la vie qui m'est sauve»), que remplacent désormais sauf et sauf que ;

2. sinon et sinon que, si ce n'est...;

3. hors, hors de, hors que, hormis, hormis que;

4. excepté, excepté que, à l'exception de ; puis, en vrac, réservé et à la réserve de, à l'exclusion de, à moins que, à $x$ ou y près... 
8 À part requerrait même une attention spéciale du fait que la locution à base nominale, sous-tendant l'interprétation « cacher » ou « trier » dans mettre à part, s'est conservée au théâtre au sens de "sans parler» et devient une préposition (dans «Mais à part ça, madame la Marquise... »), mais une curieuse préposition... postposable en dépit de Littré: un ancien raillerie à part dans le Dictionnaire de l'Académie (édition de 1694) et les modernes blague à part ou Mysogynie à part (le titre d'une chanson de George Brassens).

Une omission m'étonne chez G. Moignet. Celle de n'était. Cette petite sous-phrase pose bien des énigmes. Elle semble au début calquer une tournure latine (un exemple de Tite Live: "Pons Sublicius iter paene hostibus dedit ni unus vir fuisset " 'le pont Sublicius faillit livrer passage aux ennemis qui l'auraient franchi n'eût été un seul homme'). Le Bon Usage de M. Grevisse-A. Goosse affirme $\left(2011^{15}, \S 1159, \mathrm{e}, 2^{\circ}\right)$ qu'elle équivaut à s'il n'y avait et qu'elle concerne comme la sous-phrase en si le présent ou le futur: "N'étaient ces malheureuses jambes insensibles et inertes, je me croirais à peine en danger" (Bernanos), etc.; mais le passé également: «Il avoua plus tard [...] que plusieurs fois, n'étaient ses sentiments religieux, il se serait jeté dans la Seine" (Balzac), là où la conjonction si demanderait un plus-que-parfait s'il n'y avait eu, exceptionnel dans notre tour : «Cette déprimante monotonie, qui nous eût démoralisés n'avait été le rayonnement de l'été » (exemple d'un certain Léonce Bourliaguet, prolifique écrivain pour la jeunesse jusque-là inconnu à mon bataillon). Un subjonctif plus-que-parfait paraitrait plus normal et se rencontre d'ailleurs fréquemment : « N'eût été la fraîcheur de l'air, on se serait cru encore au mois d'août " (Butor), sans écarter complètement le subjonctif imparfait : «Le Paris torride et vaste du 15 août [...] qui serait tout à fait vidé d'humains, ne fussent quelques hordes de touristes... (Simon) et, plus étonnant, le conditionnel présent : « La tranquillité du lieu est divine - ne serait le bruit des marteaux, des rabots et des scies» (Daninos).

10 Voilà qui nous rapproche du titre emblématique de notre colloque : Si j'aurais su, j'aurais pas venu, la phrase porteuse d'une double infraction - le conditionnel derrière si et l'auxiliaire avoir conjuguant le verbe venir-, rendue célèbre par le personnage du Petit Gibus dans le film La Guerre des boutons d'Yves Robert (mais qui ne figure pas, sauf erreur, dans le roman de Louis Pergaud).

11 Les gens de ma génération n'ont pas oublié l'antienne de leurs instituteurs : « Après si, pas de -rais ». Il eût fallu préciser que seul le si hypothétique était visé, car l'interdit a paru si puissant à d'aucuns qu'un jour j'ai eu la surprise de trouver un conditionnel que j'avais utilisé en interrogation dite " indirecte » (du type - je ne me rappelle plus exactement la phrase - On s'était demandé s'il viendrait) sanctionné et assorti dans la marge d'un point d'exclamation par le relecteur d'un article soumis à une revue de linguistique.

Les déviations enfantines ou populaires pourraient se revendiquer pourtant de modèles illustres. Rappelez-vous un passage de Phèdre, mis par Racine dans la bouche de la volcanique belle-mère du chaste Hippolyte.

Voilà mon cœur : c'est là que ta main doit frapper.

Impatient déjà d'expier son offense,

Au-devant de ton bras je le sens qui s'avance.

Frappe : ou si tu le crois indigne de tes coups,

Si ta haine m'envie un supplice si doux,

Ou si d'un sang trop vil ta main serait trempée,

Au défaut de ton bras prête-moi ton épée... 

l'ordre chronologique des productions :

- l'IMP « anticipatif » (l'effet que C. Buridant a plaisamment nommé « Zorro », attesté depuis le XIV ${ }^{\mathrm{e}}$ siècle, par exemple dans la Vie de saint Louis : "Tuit estoient perdu, se ce ne fust li cuens d'Anjou " 'tous étaient perdus s'il n'y avait eu le comte d'Anjou', ou dans Le Roman de Renart : « Or estoit bien Renart cheü, / Se Diex li eüst porveü » 'Renart était hors de danger si Dieu l'avait aidé), dont J. Damourette et É. Pichon fournissent un impressionnant lot de témoignages modernes (\$1740), comme celui-ci, dû à Courteline : "Une seconde de plus, le coup partait ; je lui logeais une balle dans la peau !» = "le coup serait parti et je lui aurais logé une balle";

- l'IMP « hypocoristique » (baptisé en 1927 par L. Tesnière - et non J. Damourette et É. Pichon ainsi que l'étymologie grecque = «caressant » le ferait soupçonner); exemple, dans Le Caporal épinglé de Perret, "C'est un bon toutou, ça, monsieur, un bon chienchien aryen, on était tout beau, mais voui »;

- l'IMP «forain » (les premiers témoignages ont été observés en 1963 par M. Cohen et par A. Thérive: «Qu'est-ce qu'elle voulait, la dame?» et «Elle désirait du beurre, la petite dame?»);

- l'IMP « ludique» ou «préludique», par exemple «Toi tu étais le gendarme et moi le voleur ", signalé d'abord en Belgique - il était déjà bien connu en italien et en espagnol mais qui (cf. Patard, 2010) se répand aujourd'hui en France.

Remettons les choses à plat :

- IMP « modal », qu'est-ce à dire ? L'IMP appartient au mode indicatif. Le conditionnel (COND) aussi (la reconnaissance d'un mode "conditionnel» autonome constitue désormais un combat d'arrière-garde). Modal ne peut donc être que l'adjectif, non pas de mode mais de modalité ;

- la modalité désigne la valeur de vérité attribuée à une proposition. Elle varie en fonction de différents paramètres, parmi lesquels le temps d'époque : le passé moins sûr que le présent ( $\mathrm{du}$ fait que la mémoire peut tromper), le futur moins sûr que le présent et le passé (l'«avenir est à Dieu », et même la prédiction de l'horloge parlante ménage la place d'un fulgurant cataclysme : "Au troisième top, il sera exactement... »), le futur du passé cumulant les incertitudes du passé et du futur. 
L'IMP et le COND ne relèvent pas non plus des mêmes temps et des mêmes aspects :

- la variable temps désigne l'orientation d'un procès par rapport au repère de l'énonciation. $\mathrm{Au}$ mode indicatif, ce repère définit le «moi-ici-maintenant» ou, en un mot, l'actualité (souvent assimilée au $T_{\circ}$ ou «présent vrai» de l'énonciateur, quoique le cas échéant antérieure ou postérieure). À côté de l'actualité A de l'énonciateur, le mode indicatif comporte une actualité dépassée A' (un mixte de «moi » et de «non-moi », de «ici» et de « là », de «maintenant » et d'« alors ») qui prend en charge le sous-système des formes en -ais (IMP et COND);

- la variable aspect désigne en miroir du temps la position de l'énonciateur par rapport au procès, soit un aspect sécant (le repère intérieur au procès) ou un aspect global (le repère extérieur au procès).

19 Au total, l'IMP combine le temps passé et l'aspect sécant. Le COND combine le temps futur du passé et l'aspect global.

Tableau 1. Formules combinatoires de temps et d'aspect

\begin{tabular}{|l|l|l|}
\hline & TEMPS & ASPECT \\
\hline IMP & Passé & Sécant \\
\hline COND & Futur du passé & Global \\
\hline
\end{tabular}

En schéma (la ligne du temps, orientée de gauche à droite, dédoublée pour la lisibilité en une demi-droite destinée à recevoir les repères $\mathrm{A}$ de l'actualité, $\mathrm{A}$ ' de l'actualité dépassée, et une demi-droite parallèle où figure les procès $C$ passé, $D$ passé par rapport à $A$ mais postérieur à $\mathrm{A}^{\prime}, \mathrm{B}$ présent, E futur) : A $\rightarrow \mathrm{A}^{\prime}<->\mathrm{C}$, et $\mathrm{A} \rightarrow \mathrm{A}^{\prime}<->\mathrm{D}-\mathrm{B}-\mathrm{E}$ (les traits d'union solidarisent en un procès futur du passé les procès $D$ passé, $B$ présent, $E$ futur).

Figure 1. Représentation schématique des formes verbales

A $^{\prime}$ A

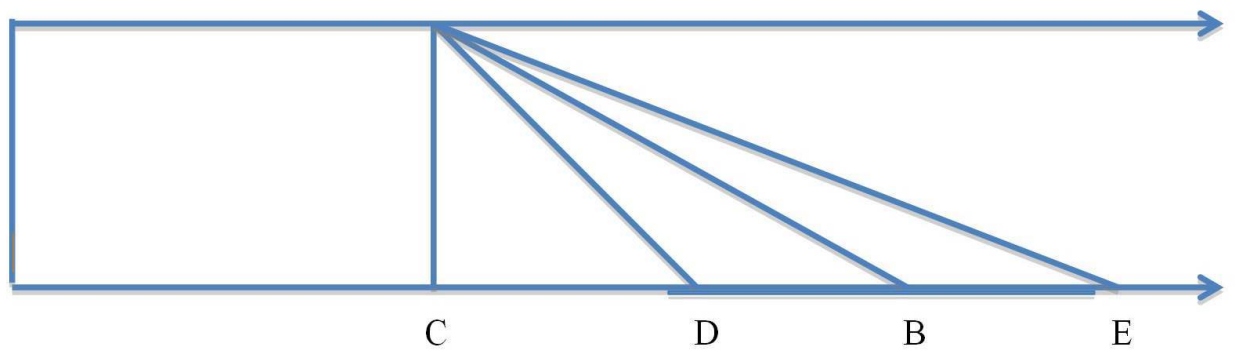

J'en arrive à la définition de l'IMP :

L'IMP EST DANS LE SYSTÈME DU VERBE LA FORME VERBALE QUI AFFIRME LA CONTEMPORANÉITÉ D'UN PROCÈS À UNE ACTUALITÉ DÉPASSÉE ET/OU L'INCLUSION DE L'ACTUALITÉ DÉPASSÉE À CE PROCÈS.

Nous n'avons plus qu'à décrypter et à exploiter l'alternative et/ou. 
- PRES - veut passablement brutal) ou « qu'est-ce qu'elle voudrait aujourd'hui, la petite dame?" (le COND voudrait lénifie, mais le temps futur du passé ouvre un délai de réaction: comparer Vous auriez l'heure? = "rien ne presse »). Dans une ambiance de marché, l'IMP voulait a la double vertu de manifester le zèle du marchand - un souhait exaucé aussitôt qu'émis - et de calmer l'impatience des clients potentiels, peu disposés à attendre le résultat des réflexions de la « petite dame » désignée à leur attention.

[5] « Toi tu étais le gendarme et moi le voleur.» 
30 À l'encontre de «toi tu serais le gendarme et moi le voleur», suggérant un jeu que l'interlocuteur garde le loisir de refuser, l'IMP étais, moins " préludique » que « ludique », considère que la distribution des rôles est d'emblée acquise.

31 Jusqu'ici, le choix de l'IMP était libre. Deux hypothèques subsistent lorsque l'aspect sécant se révèle non seulement stylistiquement recherché (exemples 1 à 5) mais presque imposé (exemple 6 en "subordonnée conditionnelle») ou carrément indispensable (exemple 7 « hypocoristique »).

[6] «Si j'étais riche, je m'achèterais une Rolls».

Vérifions que la suppression de la conjonction si ou son échange ramènent le COND : Je serais riche, je m'achèterais une Rolls ou Au cas où je serais riche, je m'achèterais une Rolls. Le morphème si aurait donc la propriété de conférer à l'hypothèse un début de réalisation afin que la conséquence puisse en découler. Il imposerait à cet effet l'aspect sécant. L'explication vaut en parallèle pour l'emploi du PRES à la place du FS («Si un jour je deviens riche, je m'achèterai une Rolls »), soit l'aspect sécant toujours préféré à l'aspect global, le temps futur sacrifié, et les usagers contraints de recourir à des moyens désambigüisants (par exemple le complément circonstanciel un jour).

[7] « C'est un bon toutou, ça, monsieur, un bon chienchien aryen, on était tout beau, mais voui » (Perret, Le Caporal épinglé, coll. Folio, p. 460).

Un COND reconduirait l'emploi ludique : On serait tout beau = «imaginons qu'on soit beau ", etc. Quelle explication dès lors? Les destinataires de ce genre d'IMP sont des animaux privés de parole, des enfants en bas âge, parfois des adultes infantilisés. Ils n'ont pas voix au chapitre. Le locuteur leur transmet une information grâce à la mélodie qu'il utilise (caressante ou grondante). La supercherie du dialogue est dénoncée - outre, par exemple, le "monsieur » et le " on » de troisième personne désignant respectivement l'allocutaire (au lieu de la deuxième personne) et le locuteur (au lieu de la première personne dans un exemple oral que j'avais noté en 1968 : «Toi, on allait te donner une fessée, tu vas voir » = “je vais") - par le temps futur du passé..., qui ne pourra toutefois être transmis que sous la forme de l'IMP, l'aspect sécant recevant pour mission de notifier une convention conclue " par-dessus la tête » des intéressés.

Le corollaire est que l'IMP derrière si hypothétique devrait réapparaitre, nonobstant un normativisme aveugle, quand l'aspect sécant cesse de prévaloir sur le temps futur du passé. C'est le cas dans l'exemple de Phèdre. Relisons-le :

Voilà mon cœur : c'est là que ta main doit frapper.

Impatient déjà d'expier son offense,

Au-devant de ton bras je le sens qui s'avance.

Frappe : ou si tu le crois indigne de tes coups,

Si ta haine m'envie un supplice si doux,

Ou si d'un sang trop vil ta main serait trempée,

Au défaut de ton bras prête-moi ton épée...

Mesurez l'inconvénient d'un IMP. Il s'accommoderait de l'expression du passé (Hippolyte déjà meurtrier, aux mains déjà tachées de sang). Idem chez Molière, quand Marianne, qu'Harpagon veut épouser, manifeste à Cléante (le propre fils du grigou), qu'elle aime autant qu'elle honnit le père, la répulsion que lui inspire en toute époque le mariage qui lui est proposé. Un PRES avez limiterait sa répugnance au présent étroit.

« Et moi, pour vous répondre, j'ai à vous dire que les choses sont fort égales; et que si vous auriez de la répugnance à me voir votre belle-mère, je n'en aurais pas moins, sans doute, à vous voir mon beau-fils. » 
min faisant, l'exception, ayant cessé d'en être une, ne mérite plus les foudres de la grammaire normative. La déclaration s'impose d'autant plus chez nous, en Belgique, que ce filon prescriptif a durablement occulté la réflexion linguistique pour des motifs qui tiennent à l'histoire : la création, après la défaite de Waterloo, d'une frontière politique avec la France, confirmée en 1830 au moment de la séparation d'avec les Pays-Bas, simultanément accompagnée de l'ouverture d'une frontière linguistique au nord, d'où résulte un risque d'éloignement de la norme française. Une série d'ouvrages entreprennent de combattre le danger : les Ne dites pas mais dites, Parlons mieux et autres Chasse aux belgicismes.

Javais entonné ce leitmotiv dans un de mes tout premiers articles, publié en 1969 par la revue La Linguistique, consacré à l'emploi du subjonctif derrière après que, que clouaient au pilori les puristes de tous poils, en faisant mienne une déclaration de Charles Bally (1965: 25) : "Quand un solécisme a la vie dure, quand il fait tache d'huile, il est rare qu'il soit négligeable; il doit s'expliquer par quelque cause générale ». Des confrères écrivains de l'Académie de langue et de littérature françaises - ils sont souvent sourcilleux en matière de norme - m'ont demandé d'en reparler. Je suis arrivé une nouvelle fois à la conclusion qu'avec le subjonctif, la langue française avait su inventer une solution à la fois économique et élégante,... tout en confessant une irrationalité personnelle du fait que je répugne, malgré mon analyse, à mettre autre chose qu'un indicatif derrière après que. Manifestation de soumission à la règle scolaire, digne des médecins de Molière : «-Juras (...) / Essere in omnibus / Consultationibus / Ancieni aviso, / Aut bono, / Aut mauvaiso? / (...) De non jamais te servire / De remediis aucunis, / Quam de ceux seulement doctæ facultatis (...) ? - Juro » (Le Malade imaginaire, V, troisième intermède) ? Ou, comme le remarquait malicieusement André Goosse : «J'ai connu des théologiens dont la foi n'était pas aussi solide que la doctrine qu'ils enseignaient.» Eh! bien, j'avais oublié que G. Moignet avait confessé une contradiction semblable lorsqu'il écrivait au terme de l'«Avant-propos» à sa thèse sur Les Signes de l'exception: «En passant, j'ai pu me convaincre de la légitimité d'“il n'y a pas que lui”. Mais d'où vient que je ne me sois jamais résolu à l'écrire?»

Nous voilà passés insensiblement des moyens linguistiques de l'exception à une certaine méfiance - une méfiance certaine - envers les prétendues déviations grammaticales.

Un pas de plus, et c'est la légitimité même de la notion d'exception en grammaire qui mérite d'être reconsidérée. Ma Grammaire critique n'en faisait pas mystère, qui proclamait dès l'«Avant-propos" (j'aurais pu ou dû mettre la maxime en épigraphe) que «l'exception infirme la règle», prenant donc à contrepied le fameux adage selon lequel «l'exception confirme la règle » ${ }^{1}$.

Je parlais d' "éthique » dans mon titre. C'est peut-être un grand mot. « Vigilance » serait plus approprié, à l'endroit notamment des idées trop bien établies pour qu'on croie nécessaire de les remettre en question. Je prendrai deux exemples en guise d'illustration conclusive, le premier relatif aux pièges de la nomenclature, le second aux contenus trop consensuels.

41 (1) Vous connaissez comme moi les discussions auxquelles ont donné lieu le nom et la notion de thème, que concurrencent sujet, topique ou foyer. P. Siblot (1998) en recensait sept termes antagonistes : rhème, prédicat, prédicat psychologique, noyau, focus, commentaire, propos. 
La plupart des auteurs ont voulu donner à thème un sens proche de l'acception courante " ce dont il est question ». Or, thème vient du grec théma, qui ne signifiait rien d'autre (une précision que je dois à notre infortuné Ivan Evrard) que "soubassement ». Dans La pluie tombe ou Il pleut des cordes, le syntagme nominal (SN) la pluie et le pronom il sont les thèmes d'une prédication dont le verbe tombe et le syntagme verbal (SV) pleut des cordes sont les rhèmes respectifs. La pluie et il sont également sujets mais deux sujets de types différents : il est seulement sujet grammatical ; la pluie est à la fois sujet grammatical et sujet logique ; des cordes est sujet logique et à la fois complément premier du verbe pleut.

Il existe deux autres types de sujets : $1^{\circ}$ le sujet sémantique (ou l'acteur d'un procès) ; $2^{\circ}$ le sujet psychologique (ce dont il est traité). La focalisation confronte le sujet psychologique au sujet logique (p. ex. C'est Clovis qui a cassé le vase de Soissons, où Clovis est à la fois sujet grammatical, sujet logique, sujet sémantique et repoussoir des sujets psychologiques autres que Clovis) et la topicalisation confronte les quatre espèces de sujets au titre des voix $1^{\circ}$ active (sujet grammatical + sujet logique \pm sujet sémantique), $2^{\circ}$ passive (sujet grammatical + sujet logique - sujet sémantique), $3^{\circ}$ moyenne (sujet grammatical + sujet logique \pm sujet sémantique + patient), $4^{\circ}$ impersonnelle (sujet grammatical - sujet logique - sujet sémantique), $5^{\circ}$ factitive (sujet grammatical + sujet logique + sujet sémantique).

(2) L'article de, avant-dernier né dans la liste des articles (le dernier identifié est l'article $\varnothing)$, a eu et a encore du mal à se démarquer de la préposition homonyme. Les grammairiens admettent généralement qu'il puisse s'associer à l'article LE pour former les « partitifs » du, de la, des. Ils imputent alors dans leur quasi-totalité le de qui alterne avec les précédents dans par exemple boire de bons vins, boire beaucoup de vin, ne pas boire de vin, à une « réduction ", à une " amputation » ou à un « effacement » de le, la, les.

est mal posé. En fait, l'article de s'allie là à l'article $\emptyset$. Dans une vision historique du système français, il faudra se demander non pas pourquoi les articles $d u$, de la, des auraient perdu leur composante le, la, les, mais pour quelle raison l'article $\varnothing$ conservateur (qui survit en français devant les noms propres de personnes et de villes: Isabelle, Bruxelles...) a fait pièce au développement de le, la, les, tous articles extensifs, c'està-dire qui visent à calquer du (-) au (+) l'extensité, ou la quantité d'êtres du monde auxquels un noyau nominal (NN) est appliqué, sur l'extension ou l'ensemble des êtres du monde auquel le NN ou un groupe nominal (GN) est applicable. On comprend que dans les trois contextes préalablement définis la démarche est inverse : boire de bons vins, beaucoup de vin ou pas de vin ont en commun de réduire l'extension du NN vin ou du GN bons vins, donc de cheminer - CQFD - du (+) au (-).

Conclusion brève et encourageante. Notre si vieille discipline (pensez qu'elle remonte à Platon et à Aristote) devrait avoir encore de beaux jours devant elle.

\section{BIBLIOGRAPHIE}

BALLY, C. (1965) ${ }^{4}$. Linguistique générale et linguistique française. Berne : Francke. 
BRES, J. (2003). « Mais oui, il était un gentil imparfait comme les autres, mon petit hypocoristique! ». Langue Française 138, p. 111-125.

BURIDANT, C. (2000). Grammaire nouvelle de l'ancien français. Paris, SEDES.

COHEN, M. (1963). Nouveaux regards sur la langue française, Paris, Éditions sociales.

DAMOURETTE, J. \& Pichon, É. (1936). Des mots à la pensée. Essai de grammaire de la langue française, vol. V. Paris : D’Artrey.

GREVISSE, M. \& GoosSE, A. (2011) $)^{15}$. Le Bon Usage. Grammaire française. Bruxelles, De Boeck.

LEEMAN, D., (2001). « Pourquoi ne peut-on combiner si et le conditionnel ? ». In Dendale, P. \& Tasmowski, L. (éds), Le Conditionnel en français. Paris : Klincksieck, p. 211-230.

LEMARÉCHAL, A. (1989). Les Parties du discours. Sémantique et syntaxe. Paris : Presses universitaires de France.

LE GOFFIC, P. (1993). Grammaire de la phrase française. Paris : Hachette.

MoIGNet, G. (1973) ${ }^{2}$. Les Signes de l'exception dans l'histoire du français. Genève : Droz.

OLIVET, T. d' (1171). Remarques sur la langue française. Paris : Huart.

PATARD, A. (2010). « L'emploi préludique de l'imparfait entre temporalité et modalité : éléments d'analyse à partir d'une étude de cas ». Journal of French Language Studies 20, p. 189-211.

ROIG, A. \& ROSIER, L. (2013). « Rien de nouveau... mis à part plusieurs pensées futiles... ». In Dufays L. \& Gournay, L. (éds), Benveniste après un demi-siècle. Regards sur l'énonciation aujourd'hui. Paris, Ophrys, p. 227-244.

SIBLOT, P., (1998). "Variations sur un thème qui “toujours déjà" prédique ». Cahiers de Praxématique 30, p. 37-53.

TESNIÈRE, L., (1927). « L'emploi des temps en français ». Bulletin de la Faculté des Lettres de Strasbourg , hors-série, p. 39-60.

THÉRIVE, A. (1963). « Petits mystères ». Carrefour (rubrique Clinique du langage), 20 nov., p. 20.

WILMET, M. (2010) $)^{5}$. Grammaire critique du français. Bruxelles : Duculot.

- (2010). « Ne me laisserez-vous que cette confusion du soir - Après que vous m'ayez, un si long jour, nourri du sel de votre solitude? (Saint-John Perse). Retour sur un subjonctif contesté ». Bulletin de l'Académie royale de langue et de littérature françaises 88, p. 107-119.

- (2011). « Temps et aspect 2 x 20 ans après ». In Duval, F. (dir.), La "logique » du sens. Autour des propositions de Robert Martin. Metz : Celted/Université de Lorraine, coll. Recherches linguistiques, p. 71-85.

\section{NOTES}

*. Texte de l'exposé oral.

1. Je crois que le coupable originel fut l'abbé d'olivet (1767:216) quand, dans une formulation moins caricaturale, soyons honnêtes, il écrit ceci à propos du participe passé invariable en construction impersonnelle: "Une exception de cette nature étant seule, et si connue de tout le monde, n'est propre qu'à confirmer notre règle, et qu'à lui affirmer de plus en plus le titre de règle générale. » 


\section{RÉSUMÉS}

Les notions d'exception et d'exclusion sont voisines. Plus exactement, la seconde découle de la première (dans une phrase comme Pierre n'aime que Marie, l'exception amoureuse que Pierre consent en faveur d'une personne se double de l'exclusion de toutes les autres). Après avoir examiné le mécanisme de la négation exceptive ne... que, le présent article envisage quelques problèmes connexes et suggère de nouvelles pistes de recherche. La sous-phrase n'était = «s'il n'y avait » fournit à ce stade l'occasion de réinterroger les motifs pour lesquels la forme en -rait est normativement interdite derrière un si hypothétique et, par contrecoup, de légitimer certaines transgressions. Du particulier au général, c'est une éthique de la discipline grammaticale, faite de souplesse pratique et de rigueur théorique, qui est défendue.

The concepts of exception and exclusion are similar. More precisely, the second ensues from the first (in a sentence like Peter only loves Mary, where the love exception granted by Pierre to some person is doubled by the exclusion of any other one). After having considered the mechanism of the exceptive negation ne... que, this article views some related problems and suggests new tracks of inquiry. The sub-sentence n'était = « if there was not » provides at this stage an opportunity for returning to the reasons why the form in -rait is normatively forbidden after a hypothetical $s i$ and, as an indirect consequence, for justifying some transgressions. From the particular to the general, it is an ethics of the grammatical discipline, made of practical flexibility and theoretical rigor, which is defended.

INDEX

Mots-clés : exception, exclusion, conditionnel, subjonctif, thème et rhème, article partitif Keywords : exception, exclusion, conditional, subjunctive, theme and rheme, partitive article

\section{AUTEUR}

\section{MARC WILMET}

Université libre de Bruxelles 